\title{
On the Privilege of Being a Diversity Scholar and How We Choose to Use It
}

\author{
Keren Dali
}

Keywords: diversity management; ethics; hiring; organizational inclusion; privilege; status; values-based diversity

Publication Type: editorial

\section{Editorial}

his editorial opens up a series of conversations that IJIDI would like to have with its readers
and authors. Editorials will raise one or several diversity-related questions and present a
combination of informative and viewpoint pieces. Opinions in the editorials are those of
the Editor or Guest Editors, whoever authors or co-authors them. Editorials will also present a
grand-tour of the respective issues.

Examining Privilege

In the context of diversity discussion, we often operate with the concept of privilege. A seminal article by Peggy McIntosh (1989), which talks about white privilege and, to a lesser extent, male privilege, defines white privilege as "an invisible weightless knapsack of special provisions, maps, passports, codebooks visas, clothes, tools and blank checks" (p. 1). The genius of Mclntosh's concept is not only it its vividness and lucidity but also in its applicability to other contexts. Preparing this issue, I was thinking about extending her metaphor and using it to analyze and understand other types of privilege that some of us have, whether we are aware of it or not, and the ways in which we use (or choose not to use) this privilege to serve the cause and ideals of diversity. McIntosh talked about privilege in the context of demographic diversity characteristics: race and gender, in this case. These characteristics are often tied into something that we refer to as "arbitrary status hierarchies" (Nishii, 2013, p. 1768), when a higher or lower social value is automatically associated with belonging to a particular demographic group (e.g., being male or female, being a person of color or white). Arbitrary status hierarchies are different from status hierarchies based on developed competencies, expertise, skills, and alliances (e.g., Cummins, 2016), which can be social or professional. The former hierarchies are "ascribed" while the latter are "achieved" (Nishii, 2018). However, in both cases, there are certain expectations associated with the placement of individuals in these hierarchies.

\section{Diversity Scholars as Ethical Role Models}

One illustrative example is academics who focus their work primarily on diversity, inclusion, social justice, and employment equity. The expectations of these individuals, developed by students, alumni, and members of respective professional communities, are often not limited to 
knowledge, expertise, and measurable performance (e.g., teaching diversity courses, producing diversity scholarship, delivering diversity training, participating in policy change and improvement, and so on). These stakeholders and partners often expect certain ethical standards and behaviors from diversity leaders, the humanistic image, if you will, the ability to set an example, and the ability to serve as a true purveyor of change. They expect bravery and integrity, which is essential for advocacy; they expect a considerable capacity for empathy and kindness. Essentially, they expect all the qualities that make a diversity practitioner or a scholar a remarkable, outstanding human being who can make a claim to ethical leadership along with extensive expertise and achievements. Metaphorically speaking, those who claim membership in the community of diversity practitioners and scholars are ascribed a higher arbitrary status in the hierarchy of ethical and humanistic behaviors.

But what if this is not the case? What if those who take the lead in diversity, inclusion, human rights, and social justice do not practice what they preach? What if the rhetoric of their keynotes, conference panels, and published papers strikes discord with their daily behaviors? What if a high-ranking administrator, known for promoting community empowerment, is complicit in verbal aggression and race-based innuendos against their minority colleagues? What if a scholar who has made their academic capital advocating for women's rights and employment equity engages in discrimination against female employees, targeting them on the basis of family status, and in bullying other marginalized groups, like students with mental illness or addiction problems? What if an experienced policy scholar openly considers students with disabilities to be a liability in a classroom and a group that should be 'dealt with' by specially trained professionals, like social workers and counsellors, rather than by professors (meant to teach 'normal' students, obviously)? What if a program Chair does not hide her convictions that firstgeneration college students are scholastically unfit for graduate school? These are painful-toobserve but easy-to-notice expressions of overt prejudice; luckily, they are less frequent.

More common are subtle behaviors, some of which amount to much-talked-about microaggressions, while others are expressions of unconscious bias, which refers to "social stereotypes about certain groups of people that individuals form outside their own conscious awareness" (Navarro, n.d.). One example is taking volunteer help for granted and not finding the time to acknowledge the selfless communal service done by our colleagues and students, often beyond the call of duty and at the expense of their personal lives and leisure. This is one of the reasons for our inability to sustain volunteer ventures over time and the low motivation of students and colleagues to engage in service endeavors. Another one is valuing positions and affiliations above the quality and originality of work, which often results in a meritocracy sham and many high-performing scholars leaving academia for the corporate world where merit feeds into the bottom line and the imperative of market competition attracts talent and potential. One of my colleagues reflected on how she spent seven months without a job and a stable affiliation, during which all invitations for host-funded talks and presentations ceased completely, despite the fact that she continued to do the same outstanding and innovative work. With a new job and respective affiliation, the stream of invitations became steady again.

Yet another example is caring about policies more than people's feelings and emotional state. The latter are often manifested in hiring and promotion processes. The Chronicle of Higher Education and other higher education publications have been abuzz with exposés on psychologically damaging academic hiring practices, whereby damage (even in the cases of nonhiring) could be easily minimized or prevented if interviewers just acted human. There are easy questions to ask ourselves as we critically assess our departmental procedures. For example, after the hiring decision is made, is it ethical to ask for letters of reference from all candidates, 
just to have our process approved as 'fair' by the HR, when we know for certain that this candidate will not be extended an offer? Do we scrutinize our interview questions for unconscious bias that disadvantages, for example, international candidates who come from non-Western cultures in which religious and folk beliefs are an integral part of individual and collective consciousness and which are more short-term-oriented than the North American culture? Do our promotion and evaluation practices have clearly outlined criteria for what constitutes 'average' vs. 'excellent' performance so that subjectivity and bias in interpreting these categories are minimized? Do we have enough heart and humanity to follow up with our colleagues who were not hired or promoted in a certain cycle? Do we care enough to inquire about their prospects and well-being, thus leaving the door open for future collaborations, collegial communication, and continuous relationships? Or do we leave them hanging until they figure out our hiring/promotion decisions on their own, thus adding to the consternation, bad blood, and latent hostility that academia is so notorious for? Have we forgotten the agony of the unknown, tied into the hiring wait? That is to say, simple human acts, simple steps to ensure that our practices are not only fair but also gentle and considerate can go a long way, no matter what policies prescribe. Every policy has its own suggested guidelines and deadlines, but many policies are there to protect the process and the institution, not the candidate or the employee.

\section{A Healthy Psychological Climate at the Forefront of Diversity Concerns}

The above examples are directly related to diversity and social justice if we think of them broadly and consider fairness and a healthy psychological climate within the purview of diversity concerns. Hence, diversity scholars and practitioners are expected to be attuned not only to the subtleties of the human condition but also to the fact that no policy should run contrary to human decency and common courtesy. If this is the case, then policy needs to change, and diversity scholars should be the ones leading that change. This is particularly important in small, intimate fields like LIS, where people know each other and run into each other through different venues and collaborative initiatives.

Both overt and more subtle injustices that we, as diversity scholars and practitioners, dare not battle or prevent put us at odds with those who expect much from us, especially our students, junior colleagues, and members of the professional community. Actions (or non-actions) that go against the admirable rhetoric of diversity and declared values of justice may detrimentally affect those who look up to us. They can undermine "students' trust in the sincerity and integrity of messages delivered by faculty members" and "in the notions of justice, equality, and inclusion" in general; weaken "the departmental rhetoric of diversity"; damage "the relationship of role models and mentorship between students and faculty"; and challenge "collegiality and inter-departmental reciprocal support" (Dali, 2018, n.p.). That is to say, by choosing to overlook injustices in our daily contexts, or simply by not being attentive and considerate enough, we dampen our own reputation as diversity leaders and willingly relinquish our potential to make change.

\section{Privilege and Power by Virtue of Achieved and Ascribed Statuses}

That is also to say that, as diversity scholars and practitioners, we have the true power to make our field more just, fair, and human. We can do it because we have the privilege granted to us by the achieved status of an academic, having a voice and a wide audience (from students to governing bodies), and enjoying a vast amount of resources at our disposal. We also have this privilege by virtue of our ascribed status as not only intellectual but also ethical leaders. This

The International Journal of Information, Diversity, \& Inclusion, 2(3), 2018

ISSN 2574-3430, publish.lib.umd.edu/IJIDI/ 
leadership comes with the expectation that we will serve as role models, speak up against unfair policies, implement just practices, and simply act as exemplary humans. We have more power than we think; the only question is whether or not we stop to recognize this power and choose to use our privilege for the greater good, changing the fabric of our departments and workplaces on the formal and informal levels. It is not enough to direct our analytical and elocutionary skill toward administrative bodies and governments and their respective policies. We have to take care of the human factor too, locally and more broadly.

We all would like to think of ourselves as nice, sensitive, and kind people. Ethical and moral people, too. We feel touched by cinematic stories and cry over fictional characters in good books; our hearts break over news about human suffering and the deteriorating environment; we rescue animals and give them a chance at a healthy, sheltered life. We just have to make sure that we are not simultaneously dismissive, neglectful, and inconsiderate of the people in our immediate surroundings: friends, neighbors, colleagues, strangers on the street, etc.

For starters, we should stop and think: what are we not doing for others that we could have easily done using our privilege? For example, can we use our seniority and reputation to step in and advocate for a colleague who is being harassed and discriminated against or should we limit our involvement to lending a shoulder to cry on and a sympathetic ear? What do we do when we see that high-achieving women with leadership potential are passed up in hiring and promotion, compared to average-achieving women and high-achieving men, because they are penalized for not meeting the expectations of femininity? Unconscious bias leads some people to expect women to be likeable and 'smart enough,' just not too smart, and "[w]hen women take the lead and assert [them]selves, [they] go against expectations-and often face pushback from men and women" (Sandberg, 2013, para. 3). Could we not use our privilege to actively change this practice?

How brave does our privilege allow us to be?

Very brave, actually. While not foolproof, our privilege is better protected than in many other settings. We have the protection of university policies on intellectual freedom; the security of tenure and union-protected seniority; and the backing of vast networks of scholars domestically and internationally. We have all the confidence we need to be brave. To be human and kind.

And sometimes, we are not as brave and kind as we could have easily been.

I have made two lists of things that I could have changed or prevented over the years by invoking my status privilege as a diversity scholar but did not. One list enumerated engagements that I did not pursue because they required the administrative and seniority pull that I simply did not have. Another list focused on things that I did not do because I was afraid to ruffle a few feathers and to stir the pot and because I wanted to be liked more than to be fair. The former list was longer; but the latter was definitely more discouraging.

\section{Then Why Do We Not Do What We Can Do?}

I used "we" throughout this editorial as one who belongs to the category of diversity scholars and one who is also sometimes implicated in unconscious bias or the lack of consideration, contributing to injustices that could have been avoided. The above ruminations are not sanctimonious preaching; the use of "we" is bona fide and related to my longstanding interest in why some people, who are basically nice and ethical, behave unethically, sometimes without 
noticing it. We are not there to hurt anyone intentionally; so why are we not living up to the ethical standards conferred on us by our privilege as diversity scholars?

I see two major reasons. One is our self-narrative. As diversity scholars, we tend to create a narrative of ourselves, one that helps us to project our public image as fair and culturally competent social justice advocates. This narrative also allows us to feel good about who we are and what we do. But the same narrative can seamlessly transform into the "always the hero to ourselves" (Moore, 2016) mantra, which is downright dangerous. Established narratives are powerful, far-reaching, and enduring, and we become ethically complacent. We cannot imagine and fathom that we may possibly engage in unethical behaviors and unfair practices, or make insensitive and inconsiderate decisions. We become less self-critical and less introspective; we stop scrutinizing ourselves (e.g., Dana, Weber, \& Xi Kuang, 2007; Dana, Lowenstein, \& Weber, 2012; Moore, 2016). From a different angle, when someone moves in the aura of a 'social justice advocate,' this makes it incredibly difficult for others to be critical of this person, which is particularly true if the scholar in question has a higher administrative position and seniority.

In addition, we become really predictable and uninteresting as scholars. We spin the same words about social justice as we did a decade ago without noticing that our message is no longer assimilated and its persuasiveness is wearing thin; we do not take risks, we do not take chances on new ideas; we fall into the trap of political correctness; we become convenient and way too agreeable to be useful for change implementation. We do not bother anyone. We realize that it is enough to 'talk about' disruption to support the narrative of ourselves as purveyors of change; there is no need to actually 'be' disruptive insofar as we discuss change repeatedly to back our diversity stature. At the outcome, our self-narrative is floated in social and professional spaces to protect our comfortable, likeable, and eloquently framed image.

The second reason is our lack of experience in something that I call 'practical diversity,' that is our ability to educate and train others for practicing diversity in daily situations, as opposed to discussing, researching, and writing about diversity. We are much more skilled at the latter, not the former. We mistakenly think that, somehow, giving a talk about unconscious bias or discrimination enables our audience to go ahead and 'do' things differently or implement change.

This is not always the case.

There are notable exceptions, with some of our colleagues delivering training sessions in organizational diversity, bias, and microaggressions on a regular basis, one of which is Nicole $A$. Cooke's “Considering Cultural Competence: Reframing our LIS Practice and Research" workshop and another one is Bharat Mehra's "Rural Libraries in the 21st Century: Places that Serve Diverse Community Needs." But these examples are not that numerous.

For the longest time, I have felt inspired by the ideas and ideals of integrating diversity in various aspects of daily life but could not make the leap from 'thinking and writing about' to 'doing and implementing.' I was missing specific instruments, methods, and introspective techniques to assess, evaluate, analyze, and modify unfair practices and unconscious bias in daily situations. This prompted me recently to complete a Certificate in Diversity \& Inclusion from the Cornell University School of Industrial and Labor Relations, and it was a game changer.

I believe that we should be those who teach because we can also do.

Part of this doing is becoming aware of our privilege as diversity scholars and channeling this

The International Journal of Information, Diversity, \& Inclusion, 2(3), 2018

ISSN 2574-3430, publish.lib.umd.edu/IJIDI/ 
privilege to achieve meaningful change.

One of the most crucial questions though is whether or not we already have model diversity scholars in our midst, or whether we all are just 'getting there'? Role models most certainly do exist: those who live their scholarship; those who feel that no matter how much they do, there is always room for self-improvement; those for whom critical and continuous reflection and introspection are part of staying current; those who remain exemplary without striving for honors and recognitions. The stance and actions of these individuals serve as an inspiration for this editorial and mark a baseline for the realization that much needed change in our treatment and practical use of scholarly privilege is imperative.

\section{The Current IJIDI Issue}

Being on the editorial team of $I J I D I$, a thriving academic journal, is part of this privilege, and it is our mission to bring to our audience both the tough and uncomfortable questions of diversity and equity policies and the in-depth humanistic and human concerns that make as much difference in people's personal lives and workplaces as regulations and formal practices. It is in this spirit of combined social and professional critique and humanistic concerns that we introduce our audience to the current July 2018 issue.

The first research article of the issue, "Rethinking Representation: Indigenous Peoples and Contexts at the University of Alberta Libraries" by Sharon Farnel et al., brings to our attention the work of the Decolonizing Description Working Group (DDWG), established at the U of Alberta, Canada, in response to "the Truth and Reconciliation Commission of Canada's Calls to Action." As part of a more encompassing effort to decolonize information access and preservation approaches related to Indigenous communities, the group was set to ensure practices that "more accurately, appropriately, and respectfully represent Indigenous peoples and contexts." Considering the sore under-representation of Indigenous concerns in the research and professional literature of LIS, IJIDI is privileged to publish this article. The reader will notice a tight connection drawn by authors between library technical services practices and the greater social, cultural, historical meaning of knowledge organization that contributes to the issues of social justice, healing, reconciliation, and future building in such multicultural countries as Canada. The article by Ahmed Alwan et al., "Microaggressions as a Barrier to Effective Collaboration Between Teaching Faculty and Academic Librarians: An Analysis of the Results of a US and Canadian Survey," gets to the heart of humanistic concerns by focusing on a healthy workplace environment in academia through an original survey of relationships between teaching faculty members and academic librarians as they interact and collaborate in the context of information literacy. Unlike many other publications that focus on microaggressions in the context of surface-level (demographic) diversity characteristics, this article looks at status-based microaggressions and delves into the intricacies of human workplace communication, with many variables at play. Bobbie Bushman's "Serving Underserved Populations: Implications from a Model of Successful Services for Deaf and Hard of Hearing Children in Public Libraries" turns to one of the often-overlooked populations and introduces a way of culturally sensitive engagement with deaf and hard of hearing children. Overlooked but not unequivocally vulnerable. The article identifies both social disadvantages and inspiring strengths found in the deaf and hard of hearing community and advocates for empowerment practices and a different level of engagement with the deaf culture. Written by a faculty member and a former librarian with the practical knowledge of American Sign Language, this article will surely resonate with many readers. The article by Ana Ndumu and Crystal Betts-Green brings the issue home, so to speak, to the crucial

The International Journal of Information, Diversity, \& Inclusion, 2(3), 2018

ISSN 2574-3430, publish.lib.umd.edu/IJIDI/ 
point where professional diversity training often begins-LIS university departments. The authors explore the diversity-related content of departmental websites, noting successes in conveying the nature of faculty diversity research and relevant funding, and shortcomings in creating diversity mission statements and displaying information on diversity initiatives and courses. In a way, the authors try to interrogate the policy-related and human dimensions of diversity in academia through their reflections on departmental websites.

The issue continues with two special section pieces which, once again, present a perfect blend of the policy-related and human factors. "NNELS: A New Model for Accessible Library Service in Canada" by Kim Johnson introduces a "user-driven, participatory, and publicly-owned accessible format collection-building" model implemented by the Canadian National Network for Equitable Library Service (NNELS). The closing piece by Deborah Schamuhn Kirk ("Driving Change: Creating a Policy Brief to Position the School Library as a Hub for Global Citizenship") reflects on the experience of an LIS Master student in creating a policy brief which focuses on the potential of "school libraries to become key players in promoting peaceful societies."

Starting with this issue, IJIDI will be featuring a book review section under the apt guidance of our new book review editor Norda Bell. The current selection includes the reviews of two books: R. J. Dowd's The Librarian's Guide to Homelessness: An Empathy-Driven Approach to Solving Problems, Preventing Conflict, and Serving Everyone (reviewed by Jorge Cardenas) and Teaching for Justice: Implementing Social Justice in the LIS Classroom, edited by N. A. Cooke and M. E. Sweeny (reviewed by LaVerne Gray). We will be excited to see many of our readers engage with the journal as book reviewers in the future, helping us to develop a true sense of community.

We hope that IJIDI readers enjoy our new collection of publications, guided by social and humanistic diversity considerations. As always, we welcome comments, communication, and questions, and look forward to future engagement with our audience.

\section{References}

Cummins, D. (2016). Status and dominance hierarchies. In Encyclopedia of Evolutionary Psychological Science. Retrieved from https://www.researchgate.net/publication/310468021_Status_and_dominance_hierarc hies

Dali, K. (2018). The right to be included: Ensuring the inclusive learning and work environment for people with disabilities in academia. Information \& Learning Science (forthcoming).

Dana, J., Lowenstein, G., and Weber, R. (2012). Ethical immunity: How people violate their own moral standards without feeling they are doing so. In D. De Cremer and A. E. Tenbrunsel (Eds.), Behavioral business ethics: Shaping an emerging field (pp. 197-515). New York: Routledge.

Dana, J., Weber, R. A., and Xi Kuang, J. (2007). Exploiting moral wiggle room: Experiments demonstrating an illusory preference for fairness. Economic Theory, 33(1), 67-80.

McIntosh, P. (1989). White privilege: Unpacking the invisible knapsack. Wellesley Centers for Women. Retrieved from https://nationalseedproject.org/white-privilege-unpacking- 


\section{the-invisible-knapsack}

Moore, C. (2016). Always the hero to ourselves: The role of self-deception in unethical behavior. In van Prooijen, J.-W. and van Lange, P. A. M. (Eds.), Cheating, corruption, and concealment: The roots of dishonesty. Cambridge, UK: Cambridge University Press.

Navarro, J. R. (n.d.). Unconscious bias. University of California, San Francisco, Office of Diversity \& Outreach. Retrieved from https://diversity.ucsf.edu/resources/unconscious-bias

Nishii, L. H. (2013). The benefits of climate for inclusion for gender-diverse groups. Academy of Management Journal, 56(6), 1754-1774.

Nishii, L. H. (2018). Sources of privilege in organizations and society. [Online lecture].

Sandberg, S. (2013). Facebook COO Sheryl Sandberg's top five ways for women to support women. People Magazine. Retrieved from https://people.com/humaninterest/facebook-coo-sheryl-sandbergs-top-five-ways-for-women-to-support-women/

Dr. Keren Dali's (keren.dali@alumni.utoronto.ca) research interests are in diversity and marginalized communities; relationships between LIS and Social Work; disabilities; LIS education with the focus on creativity, accreditation, and humanistic pedagogies; and reading behaviors. With the background in Social Work and LIS, Dr. Dali holds the inaugural Outstanding Instructor Award from the Faculty of Information, University of Toronto; the inaugural ALISE/Connie Van Fleet Award for Research Excellence in Public Library Services to Adults; the Outstanding Reviewer distinction and the Outstanding and Highly Commended Paper distinctions from the Emerald Literati Network Awards for Excellence. Her work has been funded by the grants from the Social Sciences \& Humanities Research Council of Canada (SSHRC) and the American Library Association, among others. She's active in ASIS\&T, ALISE, and IFLA, chairing committees for both ASIS\&T and ALISE. She is a winner (with Nadia Caidi) of the ALA David Cohen/ EMIERT Multicultural Award 2018 for the co-authored article "Diversity by Design" published in The Library Quarterly. 